\title{
Tanshinone I induces human colorectal cancer cell apoptosis: The potential roles of Aurora A-p53 and survivin-mediated signaling pathways
}

\author{
MINGJIE LU ${ }^{1}$, CHEN WANG ${ }^{2}$ and JIAN WANG ${ }^{1}$ \\ ${ }^{1}$ Department of Oncology, The First Affiliated Hospital of Nanjing Medical University, Nanjing, Jiangsu 210029; \\ ${ }^{2}$ Department of Hematology, Wuxi No. 2 People's Hospital, Wuxi, Jiangsu 214000, P.R. China
}

Received February 14, 2016; Accepted May 16, 2016

DOI: $10.3892 / \mathrm{ijo} .2016 .3565$

\begin{abstract}
Colorectal cancer (CRC) is one of the most common malignancies worldwide and a leading cause of cancer death. Despite decades of intensive investigations, effective interventional options are still limited and patient prognosis remains poor. Tanshinone I, an active compound from traditional Chinese herbal medicine Salvia miltiorrhiza Bunge, has been shown to inhibit cell growth of leukemia, lung, and breast cancers. However, whether and how Tanshinone I exerts similar effects on CRC needs to be elucidated. Tanshinone I induced CRC cell apoptosis was characterized and the roles of Aurora A-p53 and survivin-mediated pathways were analyzed in different CRC cell lines. Tanshinone I markedly inhibited CRC cell growth and induced apoptosis in CRC cells with functional p53 protein. Interestingly, Tanshinone I did not exert as much inhibitory effect on normal colon epithelial cells or CRC cells with mutant $\mathrm{p} 53$, indicating relative selectivity toward colorectal cancer cells with full presence of p53. In tse cells with wild-type p53, data showed that Tanshinone I mediated Aurora A inhibition results in p53 upregulation, which is required for cell apoptosis. In CRC cells with mutant $\mathrm{p} 53$ protein (not able to localize to the nucleus), however, Aurora A knockdown failed to induce CRC cell apoptosis. Instead, data showed that protein level of survivin decreased following Tanshinone I treatment. These observations were further substantiated by the pivotal role of survivin in Tanshinone I mediated apoptosis in CRC cells with p53 mutant. Tanshinone I, a novel natural compound, exerts significant inhibitory effect on CRC cell growth via a mechanism involving either Aurora A-p53 axis or survivin-involving mechanism depending on different intrinsic characteristics of tumor cells.
\end{abstract}

Correspondence to: Dr Jian Wang, Department of Oncology, The First Affiliated Hospital of Nanjing Medical University, 300 Guangzhou Road, Nanjing, Jiangsu 210029, P.R. China

E-mail:wjny8237@sohu.com

Key words: Tanshinone I, colorectal cancer, Aurora A, survivin, apoptosis, p53

\section{Introduction}

Colorectal cancer is a disease derived from the epithelial cells lining the colon or rectum of the gastrointestinal tract. Annually, $>1.2$ million people are diagnosed with colorectal cancer worldwide and almost half of them die as a consequence of disseminated disease (1). In 2011, it was the second most common cause of cancer in women and the third most common malignancy in men, the fourth most common cause of cancer death (2). It is more common in developed than developing countries (3). In addition to surgery, single-agent fluoropyrimidine in combination with fractionated pelvic radiation remains the standard regimen for advanced colorectal cancer treatment; however, $30-50 \%$ of patients with colorectal cancer will relapse and eventually die of their disease, though many patients respond well to adequate surgery and subsequent chemotherapy at the beginning of their treatment (4-7). In this scenario, it is imperative to discover novel chemotherapeutics for colorectal cancer with optimal efficacy and safety profile.

The Aurora kinases which include Aurora A, B and C, constitute one family of serine/threonine kinases whose activities are essential for the mitotic progression $(8,9)$. Aurora A is localized to the duplicated centrosomes and the spindle poles in mitosis. Several studies have characterized the roles of Aurora A in many processes required for building a bipolar spindle apparatus, including centrosome maturation and separation (10). Amplification of Aurora genes, as well as mRNA and protein upregulation, has been frequently reported in many human malignancies, such as prostate, breast, pancreas, and ovarian cancers (11-13). AURKA is located on chromosome $20 \mathrm{q}$, a genomic region that is frequently amplified in colorectal cancer, associated with adenoma-to-carcinoma progression, and an indicator of poor prognosis (14-21). In addition, mRNA and protein levels of Aurora A have also been found to be upregulated in colorectal cancer, as compared with normal tissues $(22,23)$. Due to the key role of Aurora A in mitotic progression and its upregulation in cancers, it has been considered as an important molecular target for cancer therapy $(24,25)$.

Tanshinone I (T1) is one of the major compounds from traditional Chinese herb Salvia miltiorrhiza Bunge (Danshen) which has been widely used for prevention and treatment of 
cardiovascular disease with minimal toxicity (26). Recently, the anticancer effect of $\mathrm{T} 1$ has been discovered in prostate, breast and lung cancers $(27,28)$. Moreover, T1 was revealed to greatly inhibit Aurora A expression at both gene and protein levels (11). Since Aurora A plays a stimulatory role in tumorigenesis and development in colorectal cancer, it is particularly interesting to characterize the effects of $\mathrm{T} 1$, an inhibitor of Aurora A kinase from plant, on the growth of colorectal cancer cell lines. Therefore, in this study, we evaluated the effect of $\mathrm{T} 1$ on colorectal cancer cells and try to dissect the underlying mechanisms.

\section{Materials and methods}

Reagents. Tanshinone I (T1) was purchased from LKT Laboratories (St. Paul, MN, USA). Tissue culture media DMEM with $4.5 \mathrm{~g} / 1$ glucose, L-glutamine, and sodium pyruvate as well as $0.25 \%$ trypsin was obtained from Mediatech, Inc. (Manassas, VA, USA). Fetal bovine serum (FBS) was purchased from Life Technologies, Inc. (Grand Island, NY, USA). Propidium iodide (PI) was purchased from Sigma (St. Louis, MO, USA). Antibodies against Aurora A, cyclin D1, CDK4, cleaved-PARP (c-PARP), cleaved-caspase-3 (c-caspase-3) were purchased from Cell Signaling (Beverly, CA, USA). Anti-Bax, anti-Bcl-2 and anti-survivin antibodies were from Abcam (Cambridge, MA, USA). Mouse anti-p53 monoclonal antibody was purchased from Novus (Littleton, CO, USA). Anti- $\beta$-actin antibody was from Santa Cruz (Santa Cruz, CA, USA).

Cell culture. The human normal colon epithelial cell line CCD-841CoN and colorectal adenocarcinoma cell lines, including SW480 and HCT116, were obtained from the American Type Culture Collection (Bethesda, MD, USA). The cells were maintained in high-glucose DMEM supplemented with $10 \%$ FBS, L-glutamine, sodium pyruvate and double antibiotics, in humidified $\mathrm{CO}_{2}$ incubator at $37^{\circ} \mathrm{C}$.

CellTiter Blue cell viability assay. CCD-841CoN, HCT116 and SW480 cells seeded into 96-well plates were exposed to vehicle $(0.1 \%$ DMSO) or different concentrations of T1 $(32,16$, $8,4,2,1$ and $0.5 \mu \mathrm{M}$ ) in $100 \mu \mathrm{l}$ media for $72 \mathrm{~h}$, respectively. Cell viability was quantified via CellTiter Blue (Promega, Madison, WI, USA) assay.

Anchorage-independent growth assay. HCT116 cells were plated in $0.35 \%$ top agarose (SeaPlaque agarose; Lonza, Verviers, Belgium) on a surface of $0.6 \%$ bottom agarose in 6 -well cell plates $(5,000$ cells per well) and treated with vehicle or $\mathrm{T} 1(1$ or $4 \mu \mathrm{M})$ in triplicates. After 3 weeks, the number of colonies in each well was evaluated by taking digital images through which colony numbers were counted.

PI staining for cell cycle analysis. HCT116 cells were plated and treated with $0.1 \%$ DMSO (veh) or two doses of $\mathrm{T} 1 \mathrm{(}(4$ and $1 \mu \mathrm{M})$ for $24 \mathrm{~h}$, respectively. Cells were collected by trypsinization and washed with PBS. Cells $\left(1 \times 10^{6}\right)$ were re-suspended in PBS and fixed by adding $1 \mathrm{ml}$ pre-chilled ethanol dropwisely. Cells were kept at $-20^{\circ} \mathrm{C}$ overnight and centrifuged at $300 \mathrm{~g}$ for $5 \mathrm{~min}$ to remove supernatant. Cells were washed twice with PBS, re-suspended in $500 \mu 1$ of PBS, stained with $50 \mu \mathrm{g} / \mathrm{ml}$ PI together with $50 \mu \mathrm{g} / \mathrm{ml}$ RNase at $37^{\circ} \mathrm{C}$ for $30 \mathrm{~min}$ in the dark and then analyzed on Gallios Flow Cytometer (Beckman Coulter, Brea, CA, USA) to check cell cycle distribution profile.

Apoptosis assay. HCT116 cells were plated and treated with DMSO (veh) or two doses of T1 (1 or $4 \mu \mathrm{M})$ for $72 \mathrm{~h}$, respectively. Briefly, floating cells were spun down in a centrifuge tube. The attached cells were trypsinized and combined with the floating cells, and then centrifuged for $5 \mathrm{~min}$ at 300 g. Cells were washed with cold PBS and re-suspended in $500 \mu \mathrm{l}$ of binding buffer. Each sample was added with $5 \mu \mathrm{l}$ of Annexin V-FITC and $5 \mu 1$ of 7-AAD reagents. The samples were stained at room temperature for $10 \mathrm{~min}$ in the dark. The samples were then analyzed using Gallios Flow Cytometer (Beckman Coulter). Acquired data were analyzed using the FlowJo software (Ashland, OR, USA).

Western blotting. Cells were cultured and collected for protein extraction. Cell lysates were prepared with RIPA buffer (Thermo Scientific, Rockford, IL, USA) with 1\% Halt Protease and Phosphatase Inhibitor Cocktail (Thermo Scientific). Protein samples were separated on 10 or $15 \%$ SDS-PAGE gels and transferred on PVDF membranes. Membranes were blocked with $5 \%$ non-fat milk in phosphate-buffered saline (PBS) containing $0.05 \%$ Tween-20 and incubated with the prescribed primary antibody overnight at $4^{\circ} \mathrm{C}$. Anti-Aurora A antibody $(1: 1,000)$, anti-p53 antibody $(1: 2,000)$, anti-survivin antibody (1:500) and other antibodies were appropriately diluted in blocking buffer. After incubation with the primary antibody, the membranes were washed 3 times with TBS-T and were then incubated with appropriate secondary antibodies for one hour at room temperature. Subsequently membranes were washed 4 times with TBS-T. Finally, membranes were incubated with SuperSignal West Pico Chemiluminescent Substrate (Thermo Scientific) and X-ray film was developed in the dark.

Real-time PCR. CCD-841CoN, HCT116 and SW480 cells were cultured and collected for RNA extraction. Total RNA was isolated by using Qiagen RNeasy Mini kit (Qiagen, Valencia, CA, USA) according to the manufacturer's instructions. To synthesize cDNA, $100 \mathrm{ng}$ random primer (Invitrogen, Carlsbad, CA, USA), $1.0 \mu \mathrm{g}$ of total RNA, $10 \mathrm{mM}$ dNTP and $200 \mathrm{U}$ of reverse transcriptase (Invitrogen) per $20 \mu \mathrm{l}$ reaction were used. PCRs were performed in duplicates in a $25-\mu 1$ final volume by using SYBR Green master mix from Qiagen (Qiagen), and the data were analyzed by calculating CT values. $\beta$-actin was used as the internal control. Three independent experiments were performed. The sequences of primers used in this study are as follows: $\beta$-actin for RT-PCR(F): 5'-GATGA GATTGGCATGGCTTT-3'; $\beta$-actin for RT-PCR(R): 5'-CACC TTCACCGTTCCAGTTT-3'; Aurora A for RT-PCR(F): 5'-CAT CTTCCAGGAGGACCACT-3'; Aurora A for RT-PCR(R): 5'-CAAAGAACTCCAAGGCTCCA-3'.

Construction of plasmids, preparation and application of lentivirus. Short hairpin RNAs targeting p53, Aurora A and survivin were constructed using pLKO.1 vector. The DNA 
fragments were synthesized (by GenScript Corporation, Nanjing, China), annealed and sub-cloned into the vector. The sense sequence of the sh-p53, sh-Aurora A and sh-survivin were: 5'-GTCCAGATGAAGCTCCCAGAA-3', 5'-GAGTCTA CCTAATTCTGGAAT-3' and 5'-CCGCATCTCTACATTCA AGAA-3', respectively. Lentiviral particles were produced from 293T cells using ViraPower Lentiviral expression kit (Invitrogen). Lenti-virus was applied on HCT116 or SW480 cells for $6 \mathrm{~h}$ before it was changed with fresh medium. After $24 \mathrm{~h}$, cells were selected with puromycin $(0.5 \mu \mathrm{g} / \mathrm{ml})$ for 3 days to enrich transducted cells. After that, cells were cultured in regular medium and used for different purposes.

Construction of plasmids, preparation and application of retrovirus. The DNA fragment coding full-length survivin was amplified from cDNA of SW480 cells and sub-cloned into pBABE-Neo vector using BamHI and EcoRI sites. The primers used in PCR are: 5'-GCGGATCCATGGGTGCCCC GACGTTG-3' and 5'-GCGAATTCCTAAGACATTGCTAA GGG-3'. The vector control or survivin-coding plasmid was transfected into Phoenix cells (Phoenix retroviral expression system). Virus was collected and applied onto target cells according to the standard protocol. Cells were selected with $\mathrm{G} 418(500 \mu \mathrm{g} / \mathrm{ml})$ for 5 days before they were used for different purposes.

Immuno-precipitation. To all the buffers used in the following protocol, proteinase inhibitors were added. Treated cells were collected and washed with cold PBS. Subsequently, cell pellete was re-suspended in lysis buffer $(150 \mathrm{mM} \mathrm{NaCl}, 50 \mathrm{mM}$ Tris-HCl, 1 mM EDTA, 0.5\% NP40, pH 8.0) and incubated for $20 \mathrm{~min}$ on ice. The mixture was centrifuged and the supernatant was transferred to new tubes. Anti-p53 antibody $(1 \mu \mathrm{g})$ or its isotope control was added to the cell lysate. The mixture was incubated at $4^{\circ} \mathrm{C}$ for $3 \mathrm{~h}$ with agitation. Subsequently, the mixture was incubated with protein $\mathrm{A} / \mathrm{G}$-conjugated beads for one hour. After washing, the proteins were obtained by boiling the beads in SDS sample buffer.

Statistics. The data produced in our experiments was entered into Graphpad Prism (5.01) and analyzed by Student's t-test and analysis of variance (ANOVA). Statistical significance was determined according to $\mathrm{P}$-values $\left[{ }^{*} \mathrm{P}<0.05 ;{ }^{* *} \mathrm{P}<0.01\right.$; ${ }^{* * *} \mathrm{P}<0.001$ in the figures]. Results are expressed as the mean $\pm \mathrm{SD}, \mathrm{n}=3$, if without specific indication.

\section{Results}

Effects of Tanshinone I treatment on viabilities of colorectal cancer cells. Since T1 exhibited inhibitory effects on breast and lung cancer (27-29), we sought to treat human colorectal cancer cell lines as well as normal colon epithelial cells with $\mathrm{T} 1$. After $72 \mathrm{~h}$ of $\mathrm{T} 1$ treatment at various concentrations, HCT116 colorectal cell with wild-type p53 protein displayed decreased viability in a dose-dependent manner. In contrast, this effect was partially ameliorated in SW480 cells and was largely abolished in CCD841 CoN cells (Fig. 1A). Consistently, we found that anchorage-independent growth of HCT116 cells in soft agarose was significantly inhibited following $\mathrm{T} 1$ treatment in a dose-dependent manner (Fig. 1B and C).

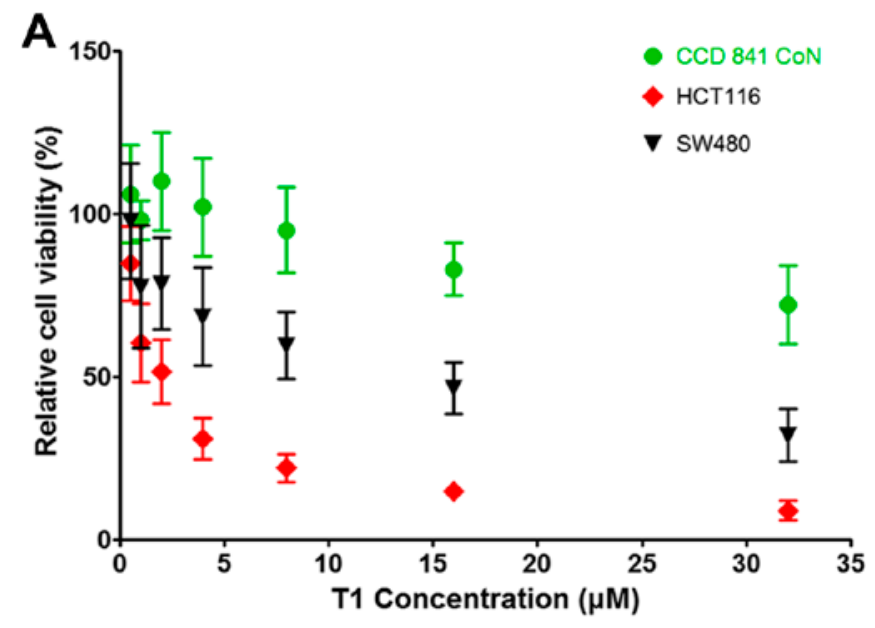

IC50: CCD 93.6 $\mu \mathrm{M}$; SW480 18.6 $\mu \mathrm{M}$; HCT116 2.16 $\mu \mathrm{M}$
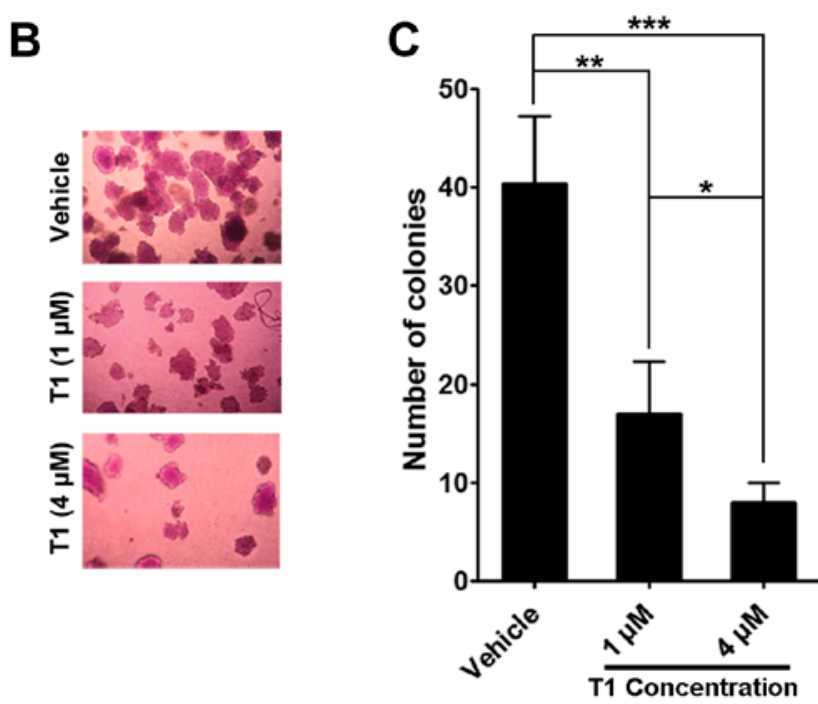

Figure 1. Growth inhibitory effect of Tanshinone I on colorectal cancer cells (A) Cell lines (CCD 841CoN, HCT116 and SW480) were treated with T1 $(0,0.5,1,2,4,8,16$ and $32 \mu \mathrm{M})$ for $72 \mathrm{~h}$ and cell viability was assessed by CellTiter Blue cell viability assay. Data indicate the mean percentage viability calculated from triplicate samples from three independent experiments $( \pm \mathrm{SD})$. (B) The effect of $\mathrm{T} 1(1$ or $4 \mu \mathrm{M})$ on anchorage-independent growth of cancer cells was measured by colony formation of HCT116 cells in soft agarose. (C) Quantification of soft-agar assay, mean $\pm S D, n=3$.

To analyze how HCT116 cancer cells were affected by T1, we conducted flow cytometric analysis to detect cell cycle profiles following T1 exposure. Results showed that when treated for $24 \mathrm{~h}$, T1 significantly increased the proportion of $\mathrm{G} 2 / \mathrm{M}$ and $4 \mathrm{~N}$ cells and decreased the proportion of $\mathrm{S}$ and G0/G1 cells (Fig. 2A), which is consistent with the downregulation of CDK4 and cyclin D1 protein levels, indicating a cell cycle arrest (Fig. 2B). Further study revealed that T1 significantly induced both early and late apoptotic events in HCT116 cells (Fig. 2C), which is consistent with the subsequent observation showing T1 induces c-PARP and Bax (both proapoptotic) protein expression as well as Bcl-2 (anti-apoptotic) downregulation in a dose-dependent manner (Fig. 2D).

The role of Aurora A and p53 in Tanshinone I mediated HCT116 cell apoptosis. As the next step, we sought to identify 
A

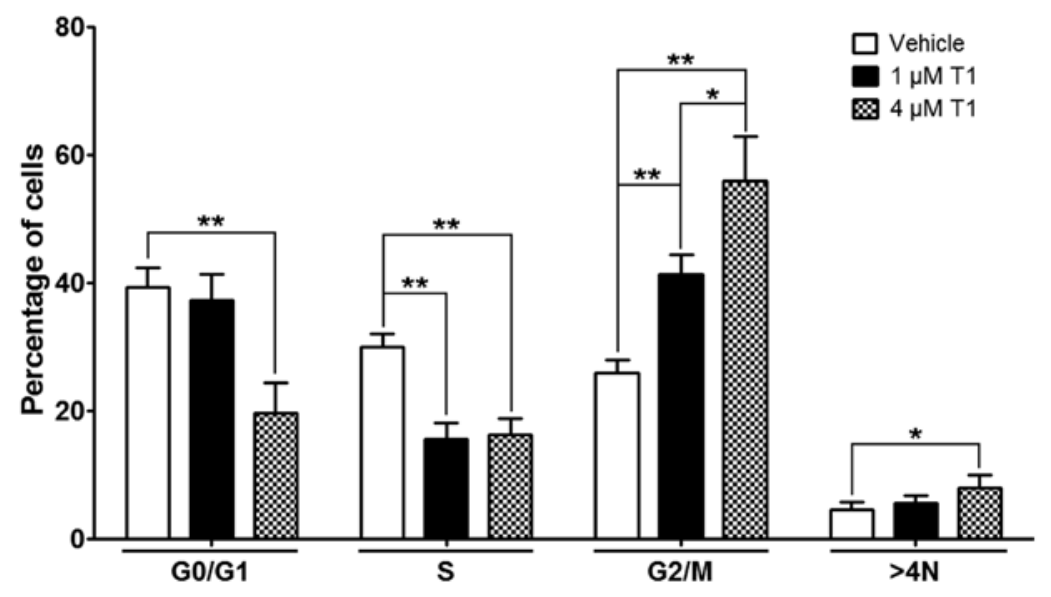

B

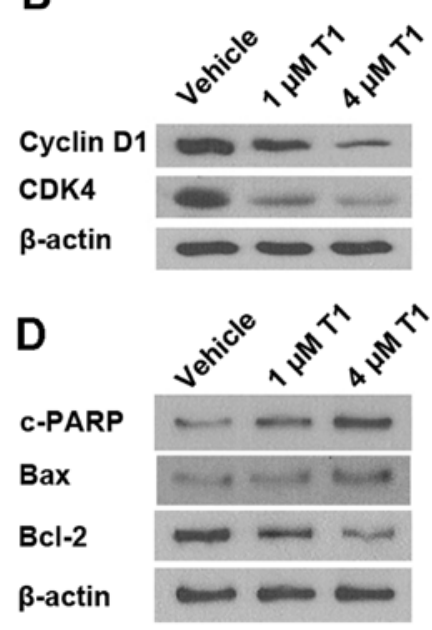

C

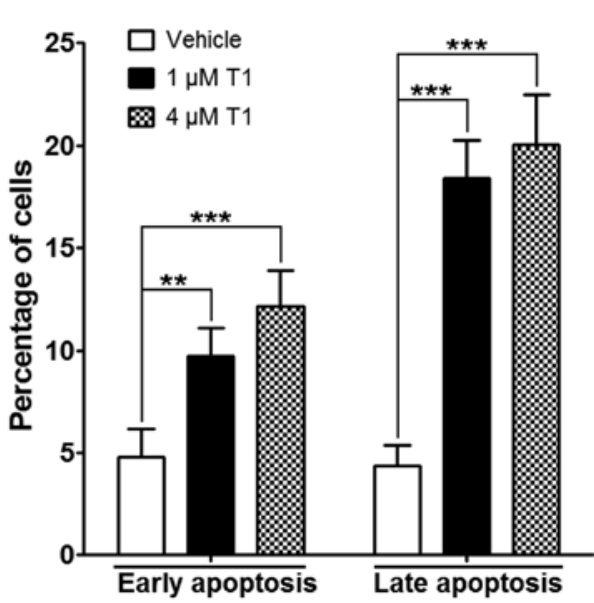

Figure 2. T1 induces cell cycle arrest and apoptosis of HCT116 cells. (A) Quantification of cell cycle assay on HCT116 cells treated with T1. (B) Protein levels of cyclin D1, CDK4 and $\beta$-actin of the above cells. (C) Quantification of FACS assay of HCT cells treated with T1; cells were analyzed for the presence of Annexin V-PE(+)/7-AAD(-) cells (early apoptosis) and Annexin V-PE (+)/7-AAD (+) cells (late apoptosis). (D) Protein levels of cleaved-PARP (c-PARP), Bax, $\mathrm{Bcl}-2$ and $\beta$-actin of the above cells.

the underlying mechanism of T1 on CRC growth and cell cycle progression. As described above, existing data show the potential roles of Aurora A on numerous malignancies other than CRC. In our experiments, we found that compared to CCD-841CoN colon epithelial cells, both HCT116 and SW480 cells exhibited significantly higher Aurora A mRNA and protein levels (Fig. 3A and B). In addition, data also revealed that T1 significantly downregulated Aurora A and phosphorylated-Aurora A protein expression in a dose-dependent manner, which seems to be strongly associated with significant p53 protein upregulation (Fig. 3C). Consistently, engineered Aurora A knock-down in HCT116 cells resulted in a similar p53 upregulation and a strong induction of apoptosis (Fig. 3D and E). Interestingly, when p53 was knocked down in HCT116 cells, Aurora A knock-down mediated apoptosis was almost completely abolished, indicating p53 is required and pivotal in this Aurora A mediated action (Fig. 3E). However, our data also suggested that p53 knock-down only partially abolished T1 mediated HCT116 cell apoptosis, indicating signaling machineries other than Aurora A-p53 pathway may exist for T1 induced apoptosis (Fig. 3F). It was reported by Katayama et al that Aurora A interacts with and phosphorylates p53, leading to its proteolysis (30). Consistent with this observation, we found that Aurora A interacted with p53 in regularly cultured HCT116 cells as shown in Fig. 3G. In addition, when the cells were treated with $\mathrm{T} 1$, the interaction between Aurora A and p53 was disrupted along with decrease of Aurora A expression (Fig. 3H).

In SW480 cells, survivin-inhibition is pivotal for Tanshinone I action. In SW480 cell line with p53 point mutation, Aurora A knock-down resulted in $\mathrm{p} 53$ protein upregulation, which is similar to data shown in Fig. 3. In contrast, we were not able to observe a similar Aurora A knock-down mediated apoptosis in SW480 cells and engineered p53 knock-down neither enhanced nor decreased cell apoptosis, indicating Aurora A-p53 axis does not play a pivotal role if mutant p53 protein is not able to localize to the nucleus (Fig. 4A and B). Further studies showed that higher concentration T1 did induce SW480 cell apoptosis (4 and $20 \mu \mathrm{m}$ ) as well as Aurora A downregulation (Fig. 4C). 
A

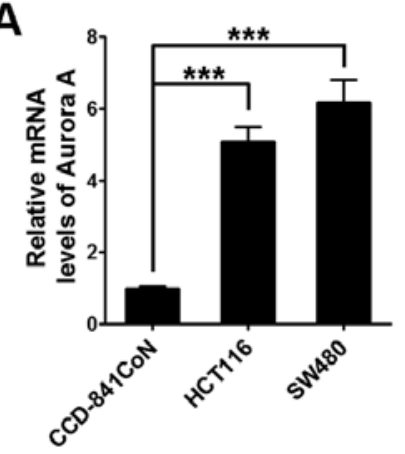

B

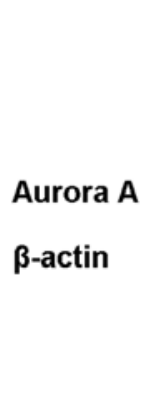

C

p53

p-Aurora A

Aurora A

$\beta$-actin
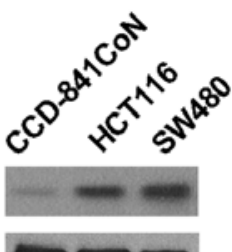

E

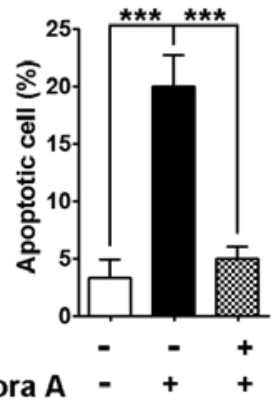

$\mathbf{F}$

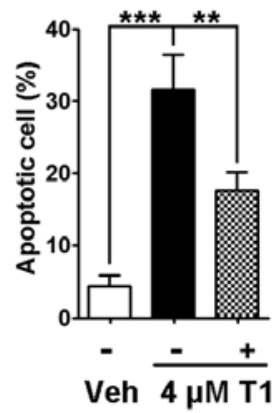

sh-p53 sh-p53

sh-Aurora A - + +
G

p53

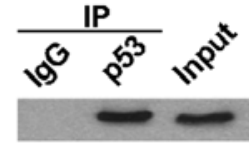

Aurora A

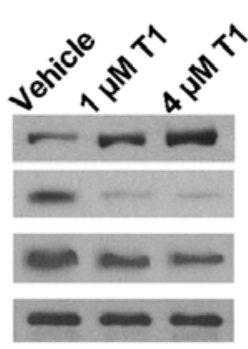

D

p53

Aurora A

$\beta$-actin

sh-p53

sh-Aurora A - + +

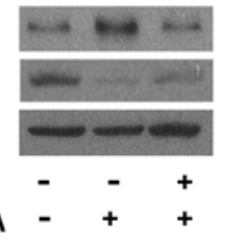

Figure 3. T1 induced apoptosis of HCT116 cells working through Aurora A-p53 pathway. (A) mRNA levels of Aurora A of different cell lines (CCD 841CoN, HCT116 and SW480). (B) Protein levels of Aurora A and $\beta$-actin of different cell lines. (C) Protein levels of Aurora A, phosphorylated-Aurora A, p53 and $\beta$-actin of HCT116 cells with or without T1 treatment. (D) Protein levels of Aurora A, p53 and $\beta$-actin of HCT116 cells with indicated treatment. (E) Quantification of FACS assay of apoptosis on cells with indicated treatment. (F) Quantification of FACS assay of apoptosis on cells with indicated treatment. (G) Immuno-precipitation assay on regularly cultured HCT116 cells using anti-p53 antibody or its isotope control. (H) Immuno-precipitation assay on HCT116 cells with or without T1 treatment.

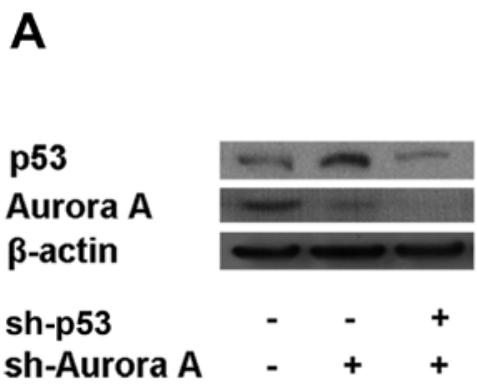

C

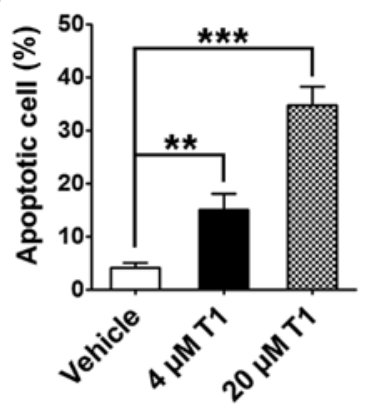

B

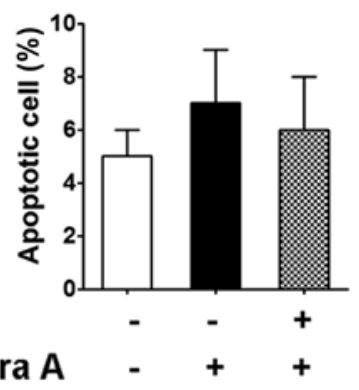

D

Survivin

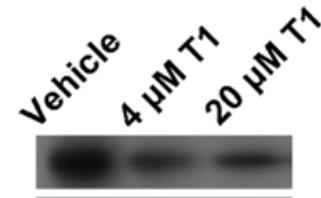

Aurora A

$\beta$-actin
H

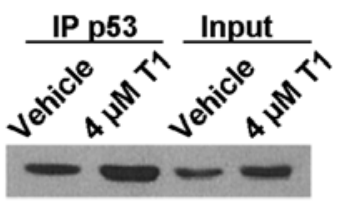

Aurora A 
A

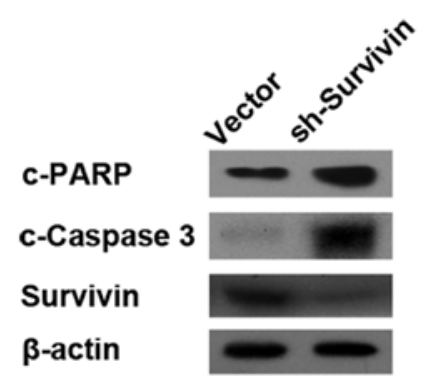

C

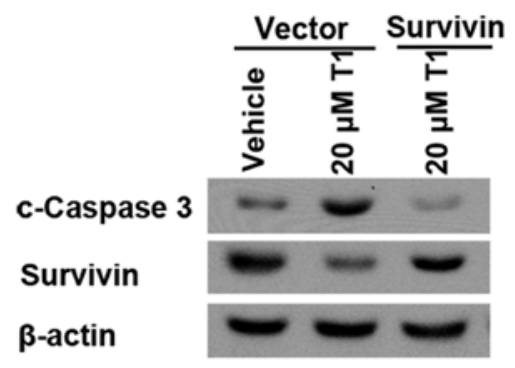

B

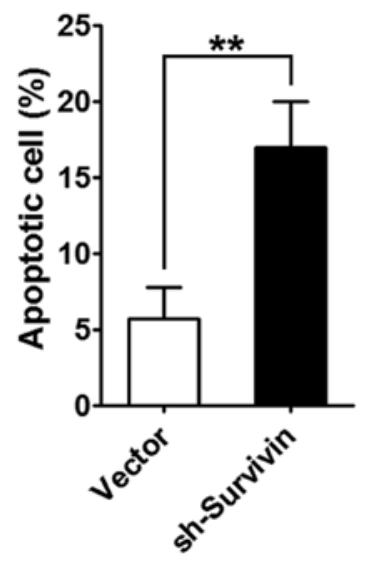

D

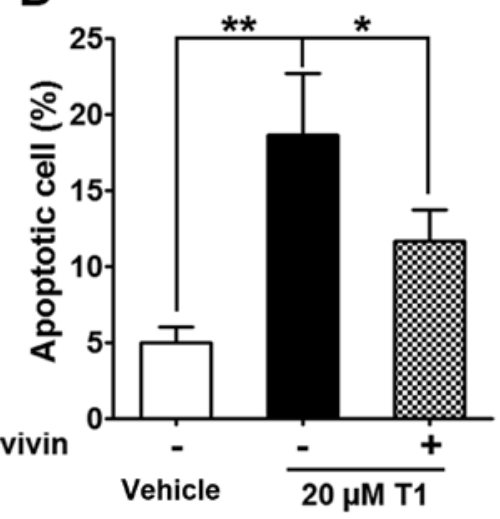

Figure 5. Survivin decrease may largely contribute to T1 induced apoptosis of SW480 cells. (A) Protein levels of cleaved-PARP (c-PARP), cleaved-caspase-3 (c-caspase-3), Survivin and $\beta$-actin of SW480 cells with or without Survivin knockdown. (B) Quantification of FACS assay of apoptosis on SW480 cells with or without Survivin knockdown. (C) Protein levels of c-caspase-3, Survivin and $\beta$-actin of SW480 cells with indicated treatment. (D) Quantification of FACS assay of apoptosis on the above cells.

c-PARP and c-caspase-3 (Fig. 5A), which is consistent with enhanced apoptosis in survivin knock-down cells (Fig. 5B). On the other hand, when SW480 cells were treated with T1, c-caspase-3 protein expression as well as SW480 apoptosis was significantly upregulated and this effect was reversed by survivin overexpression, which highlights survivin as a governor for T1 mediated SW480 cell apoptosis.

\section{Discussion}

Salvia miltiorrhiza Bunge (Danshen) is a traditional Chinese herb that has been widely adopted in the traditional Chinese medical therapy. With 20 phenolic acids, and 30 diterpene compounds, $\mathrm{T} 1$ is a relatively abundant component. In addition to the function in cardiovascular systems, tanshinones have been recently shown to possess activities against human cancers. In this study, we examined the effects of T1 on human colorectal cells in vitro. Results showed that $\mathrm{T} 1$ markedly inhibited CRC cell growth and induced apoptosis, particularly in CRC cells with functional p53 protein. Interestingly, T1 did not exert as much inhibitory effect on normal colon epithelial cells or CRC cells with mutant p53, indicating relative selectivity toward CRC cells with full presence of functional p53. In cells with wild-type p53 protein, Aurora A is critical for $\mathrm{T} 1$ action and p53 is indispensable for Aurora A-mediated signaling. In contrast, in CRC cells with mutant p53 protein (not able to localize to the nucleus), Aurora A knock-down, although it increased p53 protein level, failed to induce cell apoptosis. Instead, it seems that downregulation of survivin, an anti-apoptotic protein (31), induced apoptosis of SW480 cells following T1 treatment. These observations were further substantiated by the pivotal role of survivin in $\mathrm{T} 1$ mediated apoptosis shown in Fig. 5.

Aurora $\mathrm{A}$ is a member of the Aurora kinase family (Aurora A, B and C) (32-34). Aurora A protein overexpression and gene amplification have been frequently observed in CRC and other cancers. Bischoff et al were the first to report overexpression of Aurora A mRNA in $\mathbf{5 0 \%}$ of CRC tumors (15). It was later reported that overexpression of Aurora A protein was found in $19 \%$ of CRC samples of various disease stages by immunohistochemistry (IHC) (35). Another group identified Aurora A overexpression by IHC in $48.5 \%$ of early-stage CRC samples (23). Aurora A has been recently considered as one of the most promising molecular targets for CRC therapy (24).

In light of previous notions indicating that p53 may serve as a downstream effector for Aurora A, we hypothesized and successfully confirmed that it is also true in CRC scenario in terms of T1 action. Although we should admit that significant distinctions other than p53 protein expression exist between SW480, HCT116 and CCD-841CoN cells and p53 may not be 
the only explanation for the differential pattern of Tanshinone action observed, our argument was still strongly substantiated with a series of subsequent data from p53 knockdown cells.

P53 has been extensively implicated in regulation of cell cycle arrest, senescence and apoptosis (36). In malignant cells, higher Aurora-A level is usually associated with minimal expression of p53 (37). There are several signaling machineries which mediate Aurora-A induced p53 inactivation. First, Aurora-A phosphorylates p53 at Serine residue 315 which triggers MDM2-mediated p53 degradation (30). Alternatively, Aurora-A can also phosphorylate p53 at Serine residue 215, which disrupts its DNA binding and transactivation of downstream molecules such as p21 and PTEN (38). In addition, Nair et al showed that engineered Aurora-A silencing with siRNA promotes p53 phosphorylation at Serine residue 15, which in turn enhances G1/S cell cycle arrest (39). Furthermore, Aurora-A phosphorylates a p53 family member-p73 at Serine residue 235 and ruins physiological DNA damage-repair response (25). Taken together, it seems that Aurora-A exerts potent inhibitory effects on p53 action via phosphorylation on numerous important regulatory sites, which in turn disrupt p53-mediated innate DNA damage repair response and subsequent apoptotic events.

On the other hand, in case p53 does not function properly such as in SW480 cells, which also commonly occurred in many types of malignancies including CRC (40), our data suggested that survivin-involved pathway is responsible for T1 action.

Survivin is a member of the so-called inhibitor of apoptosis (IAP) family and exerts its regulatory effects via inhibiting caspase activation, which is required for apoptosis. Data showed that disruption of survivin induction results in increased apoptosis and decreased tumor growth. Survivin is abundantly expressed in a wide spectrum of human malignancies, but is completely absent in well differentiated cells (41). Mechanistically, survivin co-localizes with the mitotic spindle via binding to tubulin and exerts regulatory effects during mitosis. Emerging evidence suggested survivin could be regulated by p53 protein as well as Wnt pathway and $\beta$-catenin (42), indicating p53 and survivin may co-exist in the same signaling machinery. However, our data argues that survivin-involved pathway may work independently from p53-mediated pathway. In this scenario, either p53 activation or survivin inhibition may subsequently result in a similar regulatory effect.

In conclusion, our study supported that $\mathrm{T} 1$, a novel natural compound, exerts significant inhibitory effect on CRC cell growth. The underlying mechanism involves either Aurora A-p53 axis or survivin-involving mechanism depending on intrinsic characteristics of tumor cells. Although more human efficacy and safety data are needed before clinical application of $\mathrm{T} 1$ on CRC patients, our preliminary data seem to support the notion that a pre-screening for full presence of functional p53 protein in CRC tissue may be required to ensure an optimal response.

Future studies will be directed to: i) identification of the potential roles of Aurora A-p53 axis and survivin-mediated pathway in various subtypes of human CRC specimen, which highlights the significance of individualized CRC therapy and ii) screening novel herbal compounds which exert optimal inhibitory effect on CRC cell via survivin signaling.

\section{Acknowledgements}

This study was supported by intramural funding of the First Affiliated Hospital of Nanjing Medical Univeristy.

\section{References}

1. Ferlay J, Shin HR, Bray F, Forman D, Mathers C and Parkin DM: Estimates of worldwide burden of cancer in 2008: GLOBOCAN 2008. Int J Cancer 127: 2893-2917, 2010.

2. Jemal A, Bray F, Center MM, Ferlay J, Ward E and Forman D: Global cancer statistics. CA Cancer J Clin 61: 69-90, 2011.

3. Merika E, Saif MW, Katz A, Syrigos K and Morse M: Review. Colon cancer vaccines: An update. In Vivo 24: 607-628, 2010.

4. Lee YT: Local and regional recurrence of carcinoma of the colon and rectum: I. Tumour-host factors and adjuvant therapy. Surg Oncol 4: 283-293, 1995.

5. Declan Fleming RY: Colorectal cancer screening and follow-up. Surg Oncol 7: 125-137, 1998.

6. Figueredo A, Rumble RB, Maroun J, Earle CC, Cummings B, McLeod R, Zuraw L and Zwaal C; Gastrointestinal Cancer Disease Site Group of Cancer Care Ontario's Program in Evidence-based Care: Follow-up of patients with curatively resected colorectal cancer: A practice guideline. BMC Cancer 3: 26, 2003.

7. Chambers WM and Mortensen NJ: Postoperative leakage and abscess formation after colorectal surgery. Best Pract Res Clin Gastroenterol 18: 865-880, 2004.

8. Carmena M and Earnshaw WC: The cellular geography of aurora kinases. Nat Rev Mol Cell Biol 4: 842-854, 2003.

9. Nigg EA: Mitotic kinases as regulators of cell division and its checkpoints. Nat Rev Mol Cell Biol 2: 21-32, 2001.

10. Hirota T, Kunitoku N, Sasayama T, Marumoto T, Zhang D, Nitta M, Hatakeyama K and Saya H: Aurora-A and an interacting activator, the LIM protein Ajuba, are required for mitotic commitment in human cells. Cell 114: 585-598, 2003.

11. Li D, Zhu J, Firozi PF, Abbruzzese JL, Evans DB, Cleary K, Friess $\mathrm{H}$ and Sen S: Overexpression of oncogenic STK15/BTAK/ Aurora A kinase in human pancreatic cancer. Clin Cancer Res 9: 991-997, 2003.

12. Matarasso N, Bar-Shira A, Rozovski U, Rosner S and Orr-Urtreger A: Functional analysis of the Aurora Kinase A Ile31 allelic variant in human prostate. Neoplasia 9: 707-715, 2007.

13. Yamamoto S, Yamamoto-Ibusuki M, Yamamoto Y, Fujiwara S and Iwase H: A comprehensive analysis of Aurora A; transcript levels are the most reliable in association with proliferation and prognosis in breast cancer. BMC Cancer 13: 217, 2013.

14. Aust DE, Muders M, Köhler A, Schmidt M, Diebold J, Müller C, Löhrs U, Waldman FM and Baretton GB: Prognostic relevance of 20q13 gains in sporadic colorectal cancers: A FISH analysis. Scand J Gastroenterol 39: 766-772, 2004.

15. Bischoff JR, Anderson L, Zhu Y, Mossie K, Ng L, Souza B, Schryver B, Flanagan P, Clairvoyant F, Ginther C, et al: A homologue of Drosophila aurora kinase is oncogenic and amplified in human colorectal cancers. EMBO J 17: 3052-3065, 1998.

16. Carvalho B, Postma C, Mongera S, Hopmans E, Diskin S, van de Wiel MA, van Criekinge W, Thas O, Matthäi A, Cuesta MA, et al: Multiple putative oncogenes at the chromosome 20q amplicon contribute to colorectal adenoma to carcinoma progression. Gut 58: 79-89, 2009.

17. Goos JA, Coupe VM, Diosdado B, Delis-Van Diemen PM, Karga C, Beliën JA, Carvalho B, van den Tol MP, Verheul HM, Geldof AA, et al; DeCoDe PET group: Aurora kinase A (AURKA) expression in colorectal cancer liver metastasis is associated with poor prognosis. Br J Cancer 109: 2445-2452, 2013.

18. Hermsen M, Postma C, Baak J, Weiss M, Rapallo A, Sciutto A, Roemen G, Arends JW, Williams R, Giaretti W, et al: Colorectal adenoma to carcinoma progression follows multiple pathways of chromosomal instability. Gastroenterology 123: 1109-1119, 2002.

19. Nakao K, Mehta KR, Fridlyand J, Moore DH, Jain AN, Lafuente A, Wiencke JW, Terdiman JP and Waldman FM: Highresolution analysis of DNA copy number alterations in colorectal cancer by array-based comparative genomic hybridization. Carcinogenesis 25: 1345-1357, 2004. 
20. Postma C, Terwischa S, Hermsen MA, van der Sijp JR and Meijer GA: Gain of chromosome $20 \mathrm{q}$ is an indicator of poor prognosis in colorectal cancer. Cell Oncol 29: 73-75, 2007.

21. Sillars-Hardebol AH, Carvalho B, de Wit M,, Postma C, Delis-van Diemen PM, Mongera S, Ylstra B, van de Wiel MA, Meijer GA and Fijneman RJ: Identification of key genes for carcinogenic pathways associated with colorectal adenoma-tocarcinoma progression. Tumour Biol 31: 89-96, 2010

22. Gerlach U, Kayser G, Walch A, Hopt U, Schulte-Mönting J, Werner $M$ and Lassmann S: Centrosome-, chromosomalpassenger- and cell-cycle-associated mRNAs are differentially regulated in the development of sporadic colorectal cancer. J Pathol 208: 462-472, 2006.

23. Lam AK, Ong K and Ho YH: Aurora kinase expression in colorectal adenocarcinoma: Correlations with clinicopathological features, p16 expression, and telomerase activity. Hum Pathol 39: 599-604, 2008.

24. Dar AA, Goff LW, Majid S, Berlin J and El-Rifai W: Aurora kinase inhibitors - rising stars in cancer therapeutics? Mol Cancer Ther 9: 268-278, 2010.

25. Görgün G, Calabrese E, Hideshima T, Ecsedy J, Perrone G, Mani M, Ikeda H, Bianchi G, Hu Y, Cirstea D, et al: A novel Aurora-A kinase inhibitor MLN8237 induces cytotoxicity and cell-cycle arrest in multiple myeloma. Blood 115: 5202-5213, 2010.

26. Cheng TO: Cardiovascular effects of Danshen. Int J Cardiol 121: 9-22, 2007.

27. Gong Y, Li Y, Abdolmaleky HM, Li L and Zhou JR: Tanshinones inhibit the growth of breast cancer cells through epigenetic modification of Aurora A expression and function. PLoS One 7: e33656, 2012.

28. Nizamutdinova IT, Lee GW, Lee JS, Cho MK, Son KH, Jeon SJ, Kang SS, Kim YS, Lee JH, Seo HG, et al: Tanshinone I suppresses growth and invasion of human breast cancer cells, MDA-MB-231, through regulation of adhesion molecules. Carcinogenesis 29: 1885-1892, 2008.

29. Lee CY, Sher HF, Chen HW, Liu CC, Chen CH, Lin CS, Yang PC, Tsay HS and Chen JJ: Anticancer effects of tanshinone I in human non-small cell lung cancer. Mol Cancer Ther 7: 3527-3538, 2008

30. Katayama H, Sasai K, Kawai H, Yuan ZM, Bondaruk J, Suzuki F, Fujii S, Arlinghaus RB, Czerniak BA and Sen S: Phosphorylation by aurora kinase A induces Mdm2-mediated destabilization and inhibition of p53. Nat Genet 36: 55-62, 2004.

31. Tamm I, Wang Y, Sausville E, Scudiero DA, Vigna N, Oltersdorf $\mathrm{T}$ and Reed JC: IAP-family protein survivin inhibits caspase activity and apoptosis induced by Fas (CD95), Bax, caspases, and anticancer drugs. Cancer Res 58: 5315-5320, 1998
32. Giet $\mathrm{R}$ and Prigent $\mathrm{C}$ : Aurora/Ipllp-related kinases, a new oncogenic family of mitotic serine-threonine kinases. J Cell Sci 112: 3591-3601, 1999.

33. Kaestner P, Stolz A and Bastians H: Determinants for the efficiency of anticancer drugs targeting either Aurora-A or Aurora-B kinases in human colon carcinoma cells. Mol Cancer Ther 8: 2046-2056, 2009.

34. Vankayalapati H, Bearss DJ, Saldanha JW, Muñoz RM, Rojanala S, Von Hoff DD and Mahadevan D: Targeting aurora2 kinase in oncogenesis: A structural bioinformatics approach to target validation and rational drug design. Mol Cancer Ther 2: 283-294, 2003.

35. Baba Y, Nosho K, Shima K, Irahara N, Kure S, Toyoda S, Kirkner GJ, Goel A, Fuchs CS and Ogino S: Aurora-A expression is independently associated with chromosomal instability in colorectal cancer. Neoplasia 11: 418-425, 2009.

36. Wang Y, Sun H, Wang Z, Liu M, Qi Z, Meng J, Sun J and Yang G: Aurora-A: A potential DNA repair modulator. Tumour Biol 35: 2831-2836, 2014

37. Hu W, Kavanagh JJ, Deaver M, Johnston DA, Freedman RS, Verschraegen CF and Sen S: Frequent overexpression of STK15/ Aurora-A/BTAK and chromosomal instability in tumorigenic cell cultures derived from human ovarian cancer. Oncol Res 15: 49-57, 2005.

38. Liu Q, Kaneko S, Yang L, Feldman RI, Nicosia SV, Chen J and Cheng JQ: Aurora-A abrogation of p53 DNA binding and transactivation activity by phosphorylation of serine 215 . J Biol Chem 279: 52175-52182, 2004.

39. Nair JS, Ho AL and Schwartz GK: The induction of polyploidy or apoptosis by the Aurora A kinase inhibitor MK8745 is p53-dependent. Cell Cycle 11: 807-817, 2012.

40. Liu MC and Gelmann EP: P53 gene mutations: Case study of a clinical marker for solid tumors. Semin Oncol 29: 246-257, 2002.

41. Sah NK, Khan Z, Khan GJ and Bisen PS: Structural, functional and therapeutic biology of survivin. Cancer Lett 244: 164-171, 2006.

42. Olie RA, Simões-Wüst AP, Baumann B, Leech SH, Fabbro D, Stahel RA and Zangemeister-Wittke U: A novel antisense oligonucleotide targeting survivin expression induces apoptosis and sensitizes lung cancer cells to chemotherapy. Cancer Res 60: 2805-2809, 2000. 